\title{
Interchannel Interference Analysis of OFDM in a Mobile Environment ${ }^{*}$
}

\author{
Mark Russell and Gordon L. Stüber \\ School of Electrical and Computer Engineering \\ Georgia Institute of Technology \\ Atlanta, Georgia 30332-0250
}

\begin{abstract}
Othogonal frequency division multiplexing with a suitably chosen guard interval is an effective means of eliminating intersymbol interference for high-rate transmission over fading dispersive channels. Time variations of the channel, however, lead to a loss of subchannel orthogonality, resulting in interchannel interference (ICI). This article examines the effects of ICI through analysis and simulation, in the context of a system design for digital video broadcasting to mobile receivers. It is shown that ICI can be modeled as an additive Gaussian random process that leads to an error floor which can be determined analytically as a function of the Doppler frequency. Antenna diversity and trellis coding are then examined as methods for reducing this error floor.
\end{abstract}

\section{INTRODUCTION}

Orthogonal frequency division multiplexing (OFDM) is an effective technique for mitigating the effects of delay spread introduced by the mobile radio channel. OFDM uses a discrete Fourier transform (DFT) to multiplex blocks of data symbols over subchannels which are spectrally overlapping yet orthogonal in time. OFDM yields high spectral efficiency and reduces the effects of intersymbol interference (ISI) by making the block period much larger than the delay spread of the channel. The second point is especially advantageous for high bit rate applications, such as digital video broadcasting (DVB), where the channel impulse response can extend over many symbol periods. The loss of subchannel orthogonality due to interference between data blocks (ISI) can be eliminated by adding a guard interval of length greater than the maximum delay spread of the channel.

For a fading channel, however, time variations also lead to a loss of subchannel orthogonality, known as interchannel interference (ICI). If not compensated for, ICI will result in an error floor that increases with Doppler frequency. If the time-varying impulse response of the chan-

*This research was supported by the Multimedia Systems R \& D Division, Hitachi, Ltd. nel can be estimated accurately, it is possible to reduce ICI through equalization. In [3], Cimini used pilot tone assisted estimation and linear equalization before the demodulating DFT. It is also possible to use training symbol assisted estimation [7], and equalization after the DFT. Irrespective of the method, the equalizer will introduce both noise enhancement, which is especially undesirable when coding is used, and increased complexity. Furthermore, for wideband applications such as DVB, significant capacity must be sacrificed to estimate the channel with the accuracy required for effective ICI reduction. Thus the desirability of ICI equalization will depend on the acceptability of the unequalized error floor.

For a given Doppler frequency and block length, the extent to which the channel can vary during a block period decreases with increasing symbol rate. Thus, for the high rates associated with broadcasting of HDTV, systems in the literature often assume the channel does not change significantly over a block period and ignore ICI [6, 9]. At the same time, the sensitivity to errors of low bit rate HDTV decoders necessitates a bit error rate (BER) on the order of $10^{-9}$, which can make even small amounts of ICI unacceptable. This problem is further exacerbated when considering the Doppler frequencies which result from mobile reception in vehicles such as trains and buses. The purpose of this article is to analyze the degradation due to ICI, illustrating the performance limitations it generates. This will be done in the context of a DVB system for transmission of HDTV.

The system model is defined in Sect. II. In Sect. III, the origin and statistics of the ICI are analyzed and the performance of an uncoded system is discussed. The possible performance improvements resulting from antenna diversity are examined in Sect. IV, and from trellis-coded modulation (TCM) in Sect. V. Some concluding remarks are given in Sect. VI.

\section{System MODEL}

The discrete-time baseband equivalent model of the system under consideration is shown in Fig. 1. The input data $b_{s}$ consists of source coded HDTV video plus audio 


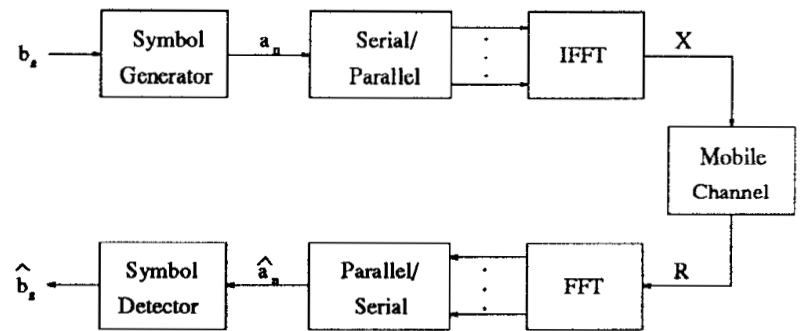

Figure 1: System model for video broadcast system using OFDM.

and control bits. Using state-of-the-art video source coding, an input rate of $20 \mathrm{Mbps}$ can be achieved [9]. In order to use the $6 \mathrm{MHz}$ bandwidth TV channels in North America, the symbol generator outputs symbols $a_{n}$ at a rate $1 / T=5$ Msps. These symbols are assumed independent, identically distributed, and possibly coded. The serial-to-parallel converter transfers blocks of symbols to the OFDM modulator, which uses an $N$-point IFFT to modulate them onto the subchannels. A guard interval of length $G$ is then added to give a transmitted sequence corresponding to samples at $t=k T_{s}$ of

$$
X_{k}^{g}=\frac{1}{\sqrt{N}} \sum_{n=0}^{N-1} a_{n} \exp \left(j \frac{2 \pi n k}{N}\right) \quad 0 \leq k \leq N+G-1
$$

where $\frac{1}{T_{s}}=\frac{1}{T}\left(1+\frac{G}{N}\right), X_{k}$ is the output sequence of the IFFT, $X_{k}^{g}=X_{(k)_{N}}$ is the sequence with guard interval, and $(k)_{N}$ is the residue of $k$ modulo $N$.

The channel is modeled as a wide sense stationary, uncorrelated scattering, Rayleigh fading channel with maximum delay $M T_{s}$. This was simulated using the 6-tap tapped-delay-line model defined in Table 1 , which represents typical values for an urban environment as given in [4]. The received sequence has the form

$$
R_{k}^{g}=\sum_{m=0}^{M-1} h_{m, k} X_{k-m}^{g} \quad 0 \leq k \leq N+G-1,
$$

where $h_{m, k}$ is the value of the channel impulse response at position $m$ and instant $k$. The demodulator removes the guard interval according to $R_{k}=R_{G+(k-G)_{N}}^{g}, 0 \leq k \leq$ $N-1$, and performs a DFT on the resulting sequence. The guard interval is set to $10 \mu s$, resulting in no ISI for the typical urban environments assumed here. Thus $X_{k-m}^{g}$ for $k<m$ corresponds to a value in the guard interval, allowing for independent analysis of the data blocks. The demodulated sequence can be written as

$Z_{l}=\sum_{n=0}^{N-1} \sum_{m=0}^{M-1} a_{n} H_{m}(n-l) \exp \left(-j \frac{2 \pi n m}{N}\right) \quad 0 \leq l \leq N-1$

\begin{tabular}{|c|c|c|c|c|c|c|}
\hline tap \# & 1 & 2 & 3 & 4 & 5 & 6 \\
\hline delay $(\mu s)$ & 0.0 & 0.2 & 0.5 & 1.6 & 2.3 & 5.0 \\
\hline frac. power & 0.19 & 0.38 & 0.24 & 0.09 & 0.06 & 0.04 \\
\hline
\end{tabular}

Table 1: 6-tap typical urban channel model, [4].

where

$$
H_{m}(n-l)=\frac{1}{N} \sum_{k=0}^{N-1} h_{m, G+(k-G)_{N}} \exp \left(j \frac{2 \pi k}{N}(n-l)\right) .
$$

The symbol detector makes decisions on the transmitted data. Since mobile reception of DVB is of interest, performance is examined as a function of Doppler frequency.

\section{ICI ANALYSIS}

It is possible to model ICI as additive interference by rewriting (3) as

$$
Z_{l}=\eta_{l} a_{l}+c_{l}
$$

where

$$
\eta_{l}=\sum_{m=0}^{M-1} H_{m}(0) \exp \left(-j \frac{2 \pi l m}{N}\right)
$$

is a multiplicative noise term and

$$
c_{l}=\sum_{n \neq l} \sum_{m=0}^{M-1} a_{n} H_{m}(n-l) \exp \left(-j \frac{2 \pi n m}{N}\right)
$$

is the random ICI term. Note that when $h_{m, k}=h_{m}$ (no time variations during block period), $H_{m}(n-l)=h_{m} \delta_{n l}$, where $\delta_{n l}$ is the Kronecker delta function, and $c_{l}=0$.

For $N$ sufficiently large, the central limit theorem can be invoked and the ICI modeled as a Gaussian random process. Since $a_{n}$ and $H_{m}(n-l)$ are independent random variables and $\mathrm{E}\left[a_{n}\right]=0, \mathrm{E}\left[c_{l}\right]=0$, where $\mathrm{E}[\cdot]$ is the expectation operator. For uncorrelated scattering and $\mathrm{E}\left[a_{n} a_{n^{\prime}}^{*}\right]=E_{s} \delta_{n n^{\prime}}$, where $E_{s}$ is the symbol energy, the autocorrelation of $c_{l}$ can be written

$$
\mathrm{E}\left[c_{l} c_{l+r}^{*}\right]=E_{s} \sum_{n \neq l, l+r} \sum_{m=0}^{M-1} \mathrm{E}\left[H_{m}(n-l) H_{m}^{*}(n-l-r)\right] .
$$

If we further assume $\sum_{m=0}^{M-1} \mathrm{E}\left[\left|h_{m, k}\right|^{2}\right]=1$ and isotropic scattering, the autocorrelation becomes

$$
\begin{aligned}
& \mathrm{E}\left[c_{l} c_{l+r}^{*}\right]=E_{s} \delta_{r}-\frac{E_{s}}{N^{2}} \sum_{k=0}^{N-1} \sum_{k=0}^{N-1} J_{0}\left(2 \pi f_{D} T_{s}\left(k-k^{\prime}\right)\right) \\
&, \cdot\left[\exp \left(j \frac{2 \pi k^{\prime} r}{N}\right)+\left(1-\delta_{r}\right) \exp \left(j \frac{2 \pi k r}{N}\right)\right](9)
\end{aligned}
$$

where $f_{D}$ is the maximum Doppler frequency and $J_{0}(\cdot)$ is the zero-order Bessel function of the first kind. Note 


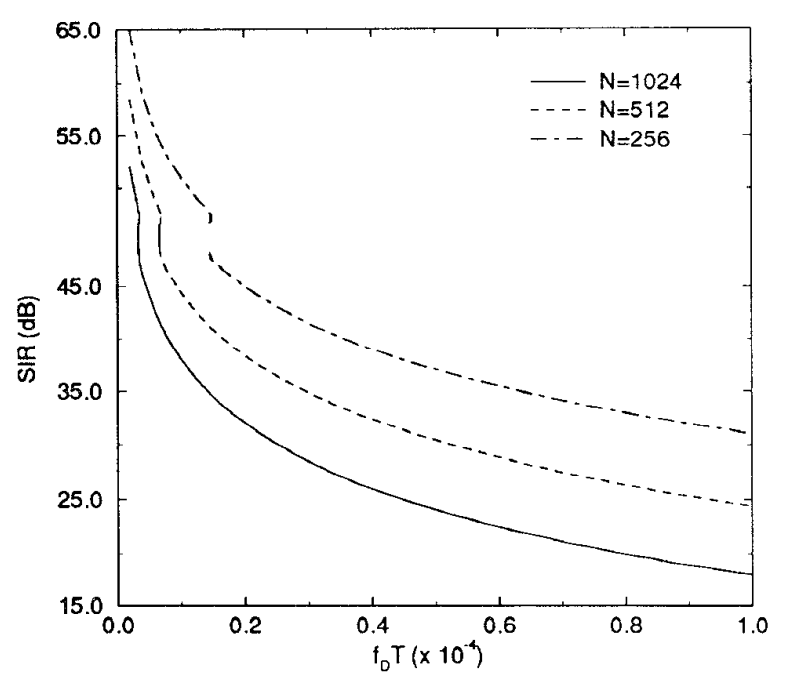

Figure 2: Signal-to-interference ratio due to ICI.

that, due to the symmetry of the summations over $k$, the autocorrelation is not influenced by the positioning of the guard interval.

For symbol-by-symbol detection, it is sufficient to examine the ICI variance, which can be simplified to give

$$
\mathrm{E}\left[\left|c_{l}\right|^{2}\right]=E_{s}-\frac{E_{s}}{N^{2}}\left\{N+2 \sum_{i=1}^{N-1}(N-i) J_{0}\left(2 \pi f_{D} T_{s} i\right)\right\}
$$

where the fact that $J_{0}(\cdot)$ is an even function has been used. Note that the degradation due to ICI is only a function of $E_{s}, N, T_{s}$, and $f_{D}$, and is independent of the signal constellation. Fig. 2 shows the ratio of symbol energy to ICI power, denoted SIR, as a function of $f_{D} T$ for several values of $N$.

For uncoded transmission of the 4 bits/symbol required for the DVB system, 16-QAM modulation is used. The symbol error rate (SER) will be used instead of the BER to simplify the analysis. The SER for 16-QAM is [8]

$$
\mathrm{SER}=3 Q\left(\sqrt{\frac{1}{5} \gamma_{s}}\right)\left[1-\frac{3}{4} Q\left(\sqrt{\frac{1}{5} \gamma_{s}}\right)\right]
$$

where $\gamma_{s}$ is the instantaneous signal-to-noise ratio (SNR) per symbol. The SER averaged over the Rayleigh fading is found by integrating (11) over the SNR distribution

$$
p\left(\gamma_{s}\right)=\frac{1}{\bar{\gamma}_{s}} \exp \left(-\gamma_{s} / \bar{\gamma}_{s}\right), \quad \gamma_{s}>0
$$

where $\overline{\gamma_{s}}$ is the average SNR. This was done numerically and, for large $\mathrm{SNR}$, the result is $\mathrm{SER} \approx 6.48 / \bar{\gamma}_{s}$. Substituting the SIR for $\bar{\gamma}_{s}$ gives the error floor due to ICI, which is shown in Fig. 3. Simulation results are also given to confirm the analysis. This result assumes perfect knowledge of $\eta_{l}$, which can be derived by estimating the channel

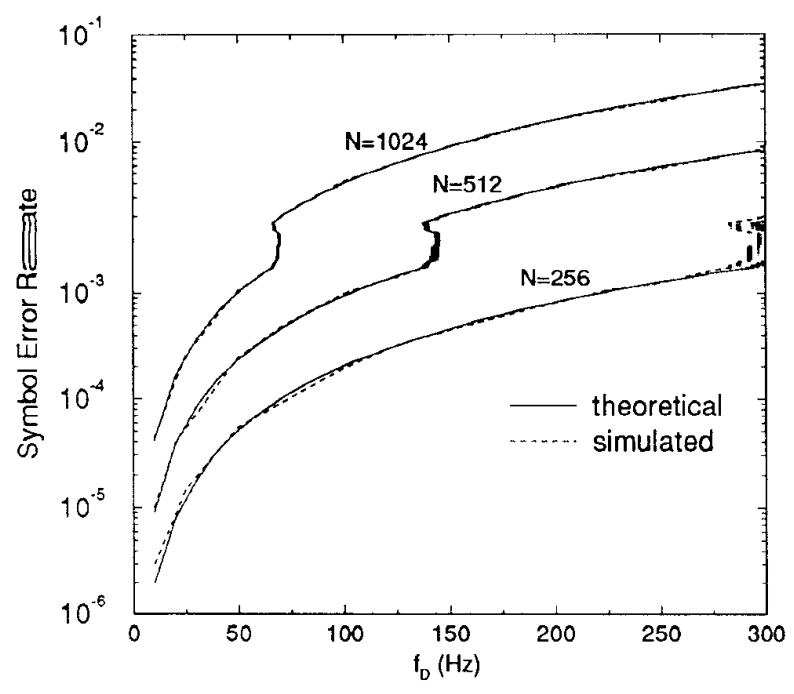

Figure 3: Error floor due to ICI for 16-QAM.

impulse response. In practice, it may be preferable to decrease complexity by sending pilot symbols and accepting an ICI corrupted version of $\eta_{l}$.

Fig. 3 illustrates that the choice of $N$ represents a trade-off between loss in capacity due to the guard interval and ICI degradation. For $N \in\{256,512,1024\}$ and $G=10 \mu \mathrm{s} / T_{s}$, the percentage of capacity lost is $\{16.8 \%, 8.9 \%, 4.7 \%\}$, respectively. Irrespective of $N$, however, even for small values of $f_{D}$, an error floor below $10^{-9}$ cannot be achieved for a fading channel without additional effort. Since fading compensation is desirable in and of itself, it is of interest to analyze the effects of fading compensation on ICI performance. The next two sections will examine the ability of two common techniques, antenna diversity and trellis-coded modulation, to provide ICI performance improvement.

\section{Antenna Diversity}

Antenna diversity is an effective means of reducing the degrading effects of a fading channel, as well as being "compatible with coding. It is thus of interest to examine the ability of antenna diversity to reduce the effects of ICI. There are a variety of signal combining methods which offer a trade-off between performance and complexity [8]. In terms of ICI performance, however, the less complex, power-based combining techniques such as selection diversity will offer no improvement. This is because ICI is not a function of signal power, but rather the rate at which the channel varies. Thus this section will consider maximum ratio combining ( $\mathrm{MRC}$ ). It will be assumed that the received signals at the various antennas are mutually uncorrelated.

MRC uses knowledge of the channel state information (CSI) and the fact that the CSI is uncorrelated with the noise to improve the received SNR. For the OFDM system 


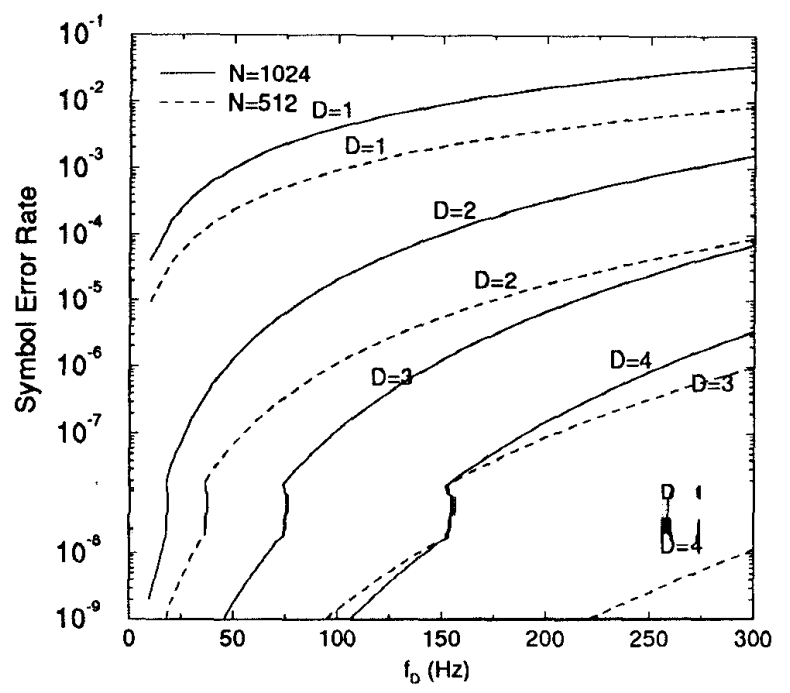

Figure 4: Error floor due to ICI for 16-QAM and antenna diversity with MRC.

using $D$ antennas, the combining will be performed after the demodulating FFT, thus requiring $D$ front-ends and $D$ OFDM demodulators. The resulting decision variable is

$$
Z_{l}=\sum_{d=1}^{D}\left|\eta_{l}^{d}\right|^{2} a_{l}+\eta_{l}^{d *} c_{l}^{d}
$$

MRC will improve ICI performance in the same way as it improves noise performance as described in [8] if $\eta_{l}^{d}$ and $c_{l}^{d}$ are uncorrelated. The prescence of the data $a_{n}$ in $c_{l}$ provides this decorrelation. The SER performance of 16QAM can be calculated by replacing the SNR distribution of (12) with

$$
p\left(\gamma_{s}\right)=\frac{1}{(D-1)\left(\bar{\gamma}_{c}\right)^{D}} \gamma_{s}^{D-1} \exp \left[-\gamma_{s} / \bar{\gamma}_{c}\right] \gamma_{s}>0
$$

where $\bar{\gamma}_{c}$ is the average SNR per diversity channel. The ICI performance can be calculated by substituting the SIR for $\bar{\gamma}_{c}$, which is shown in Fig. 4 for $N \in\{512,1024\}$ and $D \in\{1,2,3,4\}$. It can be seen that the diversity gain resulting from $\mathrm{MRC}$ can provide an acceptable error floor over a wide range of Doppler frequencies. Note that, since the rate of increase in ICI power decreases with Doppler frequency, values of $D>2$ offer a significant extension of the range of $f_{D}$ over which a SER of $10^{-9}$ can be achieved.

\section{Trellis-coded Modulation}

Channel coding is another technique for improving performance on a fading channel. Due to the bandwidth constraints imposed on DVB, the bandwidth efficiency of TCM makes it a sensible candidate. Some papers have suggested reducing the requirements on the TCM code by concatenating an outer Reed-Solomon (R.S) code. This is a reasonable suggestion for Europe, where the bandwidth of the analog TV channel is either 7 or $8 \mathrm{MHz}$. However, for a $6 \mathrm{MHz}$ bandwidth, the increase in bit rate due to RS coding would necessitate an increase in signal constellation size in order to maintain the extra bandwidth needed to absorb the guard interval, pilot symbols, adjacent channel protection, and NTSC co-channel interference rejection. Thus only TCM will be considered. The symbol generator of Fig. 1 is now considered to also contain a TCM encoder, while the symbol detector contains a Viterbi decoder. It is assumed that interleaving, which for OFDM can be done in both frequency and time, is sufficient such that successive symbols are independently

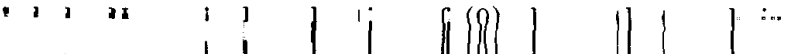
faded. Numerical evaluation of (9) shows that such interleaving is also sufficient to provide successive samples whose ICI terms are uncorrelated.

\section{V-A 8-AM Coding}

For the Rayleigh channel, the error rate decreases exponentially with the minimum error event length $l_{m}$ and linearly with the product distance of the error events. Thus $l_{m}$ is the most important design parameter. As shown in [5] for rate $n /(n+1)$ coding, as $n$ increases the value of $l_{m}$ achievable for a given constraint length $\nu$ decreases. In fact, for rate $4 / 5$ coding $\nu=9$ is required to achieve $l_{m}=3$. This is important because the complexity of the assumed Viterbi decoder increases exponentially with $\nu$.

Improved performance can be achieved by using rate $n^{\prime} /\left(n^{\prime}+1\right)$ coding with PAM modulation independently on the in-phase (I) and quadrature (Q) channels [2], where $n^{\prime}=n / 2$. For $n=4$, this gives rate $2 / 3$ coding on the I and $\mathrm{Q}$ channels, for which $l_{m}=3$ can be achieved with $\nu=4$. The resulting overall constellation is 64-QAM. The codes chosen are given in [2] and were designed for 8-PSK on the Rayleigh channel.

\section{V-B Performance Bounds}

Since ideal CSI is assumed, the SER for the hybrid, two-dimensional coding scheme can be determined from that of the one-dimensional 8-PAM coding scheme via

$$
\mathrm{SER}_{2 d}=1-\left(1-\mathrm{SER}_{1 d}\right)^{2} .
$$

An upper bound on performance was obtained using the Chernoff bound on the error event probability and a truncated union bound on the overall error probability [2]. Since the codes are uniform, the code spectrum can be determined by comparing error events versus the all zero code word and using the error weight profile for 8-PAM. The Chernoff bound is then calculated for each error event and multiplied by the number of symbol errors corresponding to that error event. Finally, an approximation on the union bound for the overall error probability is found by summing the SER probabilities due to error 


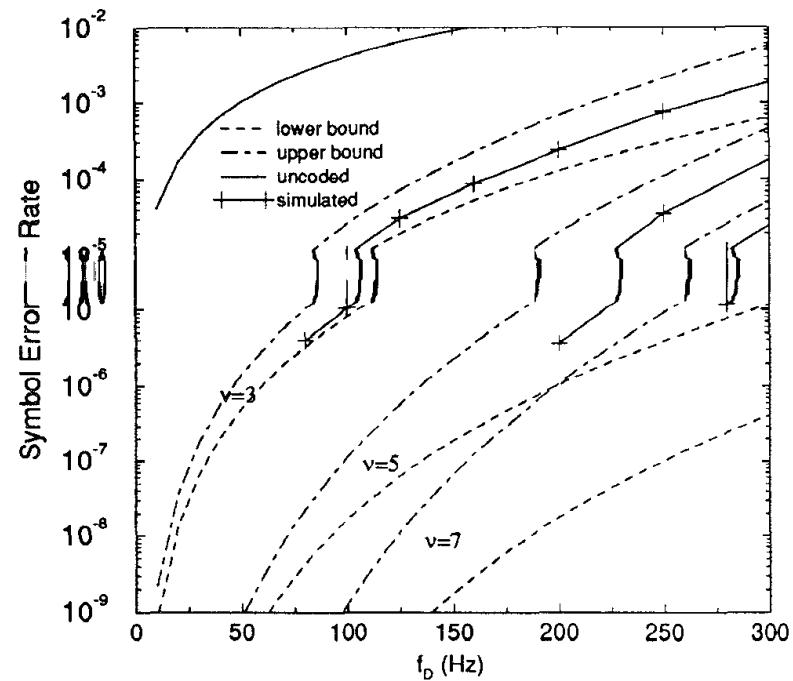

Figure 5: ICI error floor using TCM, $N=1024$.

events which have length $\leq l_{m}+3$. A lower bound on the error probability can be determined by calculating the exact error probability due to the most likely error event, as described in [1].

The bounds on ICl error floor for $\nu \in\{3,5,7\}$ are given in Fig. 5 for $N=1024$ and Fig. 6 for $N=512$. Simulation results are also given to confirm the bounds. The codes illustrated here have lower bounds that include only a single error event of length $l_{m}$. Thus the lower bound becomes tighter much more rapidly for these codes than for codes having multiple error events of length $l_{m}$. These results show that using TCM coding independently on the I and Q channels is an effective method of reducing the effects of ICI. Furthermore, for the low error rates required for DVB, moving to larger values of $\nu$ offers significantly more gain than for applications where the error rate constraints are less strict.

\section{Conclusions}

The interchannel interference resulting from the use of OFDM over a Rayleigh fading channel has been analyzed in the context of a system for transmission of HDTV to mobile receivers. It was shown that ICI leads to an error floor dependent on the number of subchannels and the fading rate. For the error rates in the range of $10^{-9}$ required by state-of-the-art video source decoders, ICI alone can lead to an unacceptable error rate.

Antenna diversity and trellis-coded modulation were examined as methods of reducing the error floor. Antenna diversity with maximum ratio combining was found to provide acceptable performance over a wide range of Doppler frequencies. This was also found to be the case for TCM. The performance improvement using rate $2 / 3$ coding independently on the $\mathrm{I}$ and $\mathrm{Q}$ chamels versus 2dimensional rate $4 / 5$ coding was discussed. Although not

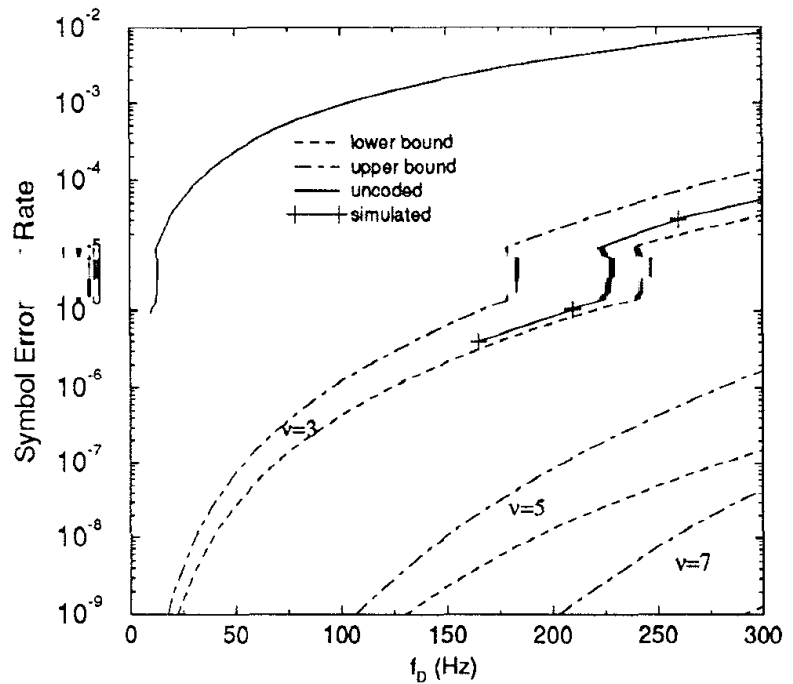

Figure 6: ICI error floor using TCM, $N=512$.

considered in this paper, it is reasonable that a combination of diversity and TCM would provide even further performance improvement.

\section{REFERENCES}

[1] E. Biglieri and P.J. McLane, "Uniform distance and error probability properties for TCM schemes," IEEE Trans. Commun., vol. 39, No. 1, pp. 41-52, Jan. 1991.

[2] A. Brajal and A. Chouly, "Optimal trellis-coded 8-PSK and 4-AM modulations for the Rayleigh channel," Proc. ICC '94, pp. 28-33, New Orleans, Dec. 1994.

[3] L.J. Cimini, Jr., "Analysis and simulation of a digital mobile channel using orthogonal frequency division multiplexing," IEEE Trans. Commun., vol. COM-33, pp. 665-675, July 1985.

[4] COST 207 Management Committee, "COST 207: digital land mobile radio communications," Commission of the European Communities, Luxembourg 1989.

[5] J. Du, Y. Kamio, H. Sasaoka, and B. Vucetic, "Trelliscoded M-QAM for efficient data transmission over land mobile radio channels," Proc. PIMRC '93, pp. C1.6.1C1.6.5, Yokohama, Sept. 1993.

[6] J.F. Helard and B. Le Floch, "Trellis coded orthogonal frequency division multiplexing for digital video transmission," Proc. GLOBECOM '91, pp. 785-91, Phoenix, Dec. 1991.

[7] P. Hoeher, "TCM on frequency-selective land-mobile fading channels," Proc. of Fifth Tirrenia International Workshop, pp. 317-28, Tirrenia, Sept. 1991.

[8] J.G. Proakis, Digital Communications, McGraw-Hill: New York, 1983.

[9] M. Sablatash, "Transmission of all-digital advanced television: state of the art and future directions," IEEE Trans. Broadcasting, vol. 40, pp. 102-121, June 1994. 